\title{
mtDNA mammalian evolution: mice walk with many little steps while elephants with a few big
}

\author{
Dmitrii Iliushchenko \\ Center for Mitochondrial Functional \\ Genomics, Immanuel Kant Baltic \\ Federal, University \\ Kaliningrad, Russia Federation \\ iliushchenkodmitrii@gmail.com
}

\author{
Anastasia Sokol \\ Center for Mitochondrial Functional \\ Genomics, Immanuel Kant Baltic \\ Federal, University \\ Kaliningrad, Russia Federation \\ anastasia3sokol@yandex.ru \\ Konstantin Gunbin \\ The Institute of Cytology and Genetics \\ of the SB RAS \\ Novosibirsk, Russia Federation \\ genkvg@gmail.com
}

\author{
Konstantin Popadin \\ School of Life Sciences \\ Ecole Polytechnique Federale de \\ Lausanne, Lausanne, Switzerland \\ Center for Mitochondrial Functional \\ Genomics, Immanuel Kant Baltic \\ Federal, University \\ Kaliningrad, Russia Federation \\ konstantinpopadin@gmail.com
}

\begin{abstract}
In this project, we use aligned mitochondrial genomes (353 species, 40 families, 13 genes) to test the hypothesis that, with a decrease in body weight, the rate of evolution will be faster due to very neutral substitutions, and with an increase in body weight there will be an increase in the rate of evolution overdue to more radical substitutions.

Keywords - mitochondrial DNA, mammalians, evolution rate
\end{abstract}

\section{Introduction}

The ecological properties of species drive different patterns of molecular evolution. Numerous associations of ecological and genetic traits are especially well known for mammalian species - which are well characterized ecologically and deeply sequenced. It is known, for example, that small-bodied short-lived (hereafter 'mice') mammalian species have a higher rate of neutral evolution (i.e. accumulation of synonymous substitutions per the unit of time) as compared to large-bodied long-lived (hereafter 'elephants') species [1]. Most likely this effect is driven by the different number of DNA replications of germ cells per unit of time (higher in mice versus elephants). Also, it is known that slightly-deleterious variants, opposite to the neutral mutations, tend to accumulate faster in elephants versus mice because of their lower effective population size which leads to stronger genetic drift. Interestingly, both these most common categories of mutations (neutral and slightly-deleterious) together very often lead to an approximately constant rate of molecular evolution: mice accumulate numerous neutral (or close to neutral) substitutions, while elephants accumulate more rarer and more deleterious variants. Here we use mitochondrial genomes to test the hypothesis that in physicochemical space "mice walk with many little steps while elephants with a few big ones".

\section{Methods}

Mitochondrial genes, which we used in this project allow us to analyze hundreds of mammalian species with a complete sequenced mtDNA and hundreds of species with at least 10 available within-species sequences. We plan to find a physicochemical metrics of mtDNA evolution to describe and analyze mice-specific and elephant-specific patterns of amino-acid substitutions.

Using codon alignment of mitochondrial genes (353 species, 40 families, 13 genes) we generated all possible trios of species within each family. For each species from each trio, we obtained generation length as the main ecological factor, associated with effective population size. The approach is based on the relative ratio test [2]: we compare two species $\mathrm{B}$ and $\mathrm{C}$ with different generation length. If species $\mathrm{B}$ is more long-lived as compared to species $\mathrm{C}$ we expect that the rate of neutral evolution will be decreased during the B-specific evolution, while the rate of accumulation of slightly-deleterious variants will be increased. To test it we use an outgroup species A to analyze the pattern of evolution between A-B and A-C. Finally, we work with different outgroups for each pair of species to demonstrate the robustness of the observed effects. Additionally to comparative species scale, we analyze genetic polymorphisms in all mammalian species with sequenced al least 10 different organisms. We expect, that populations of large-bodied mammals would demonstrate an increased frequency of slightly-deleterious variants, segregating within the population.

\section{Results}

Our pilot results from comparative-species scale demonstrate the expected trend: long-lived species tend to accumulate more distant amino-acid substitutions (estimated as increased average Grantham distance of the amino-acid substitutions) as compared to short-lived species. Currently, we use additional properties of aminoacids to find a physicochemical signature of substitutions typical for shortlived versus long-lived species. Our pilot results from the intra-species scale also demonstrate expected results: an increased frequency of more deleterious (more biochemically distant) variants segregating in the long-lived species.

\section{Discussion}

Both results emphasize relaxed purifying selection in long-lived species because of their low effective population size. Future analyses will uncover a more detail signature of amino-acid substitutions patterns in mammalian species with different generation lengths.

\section{REFERENCES}

[1] K. Popadin et al. Accumulation of slightly deleterious mutations in mitochondrial protein-coding genes of large versus small mammals, PNAS, 2007104 (33) 13390-13395; https://doi.org/10.1073/pnas.0701256104.

[2] L. Bromham, et al. The Power of Relative Rates Tests Depends on the Data, J Mol Evol (2000) 50:296-301DOI: $10.1007 / \mathrm{s} 002399910034$. 